\title{
REJEIÇÃO DE PERTURBAÇÕES POR REALIMENTAÇÃO ESTÁTICA DE SAÍDA EM SISTEMAS LINEARES
}

\author{
Carlos E. T. Dórea* \\ cetdorea@ufba.br
}

\author{
Basílio E. A. Milani ${ }^{\dagger}$ \\ basilio@dt.fee.unicamp.br
}

${ }^{*}$ Departamento de Engenharia Elétrica, Escola Politécnica, Universidade Federal da Bahia, Rua Aristides Novis 2,
40210-630 Salvador, BA, Brasil.

${ }^{\dagger}$ Faculdade de Engenharia Elétrica e de Computação, Universidade Estadual de Campinas, C.P. 6101,

13081-970 Campinas SP, Brasil.

\begin{abstract}
This work adresses the Disturbance Decoupling Problem in linear systems via static output feedback. Necessary and sufficient conditions for solvability in two important families of systems are established. The problem is solvable if and only if a given subspace verifies an invariance property. The set of output feedback matrices which solve the problem is then parameterized through a suitable change of the coordinate basis of the state, input and output spaces. A numerical example illustrates the proposed approach.
\end{abstract}

KEYWORDS: Linear systems, disturbance rejection, geometric approaches, invariance, feedback control.

\section{RESUMO}

Este trabalho estuda o problema de rejeição total de perturbações em sistemas lineares através de realimentação estática de saída. Condições necessárias e suficientes para a existência de soluções em duas importantes famílias de sistemas são estabelecidas. O problema é solúvel se e somente se um determinado subespaço goza de uma propriedade de invariância. O conjunto de matrizes de realimentação de saída que resolvem o problema é então parametrizado através de uma mudança adequada da base de representação dos espa-

\footnotetext{
Artigo submetido em 20/12/00

1a. Revisão em 04/04/02

Aceito sob recomendação do Ed. Assoc. Prof. Liu Hsu
}

ços de estado, controle e saída. Um exemplo numérico ilustra a abordagem proposta.

PALAVRAS-CHAVE: Sistemas lineares, rejeição de distúrbios, abordagem geométrica, invariância, controle por realimentação.

\section{INTRODUÇÃO}

A solução teórica do problema de rejeição de perturbações por realimentação completa de estado (DDP, do inglês Disturbance Decoupling Problem) constitui-se em um excelente exemplo ilustrativo da aplicação de alguns dos conceitos fundamentais da chamada abordagem geométrica para o controle de sistemas lineares, tais como subespaços $(A, B)$ invariantes e de controlabilidade. Condições geométricas de existência de soluções para o DDP podem ser encontradas, por exemplo, em (Wonham, 1985; Basile e Marro, 1992).

A solução do DDP (quando existente) não é única. Um problema interessante é tentar parametrizar o conjunto de todos os controladores que resolvem o DDP. Tal problema, no caso geral, é de muito difícil solução. Alguns trabalhos conseguiram no entanto resolvê-lo para alguns tipos de sistema. Como exemplo pode ser citado o artigo (Dórea e Milani, 1997), onde foram obtidas a solução completa e a parametrização de todos os controladores que resolvem o DDP para uma classe particular de sistemas.

Quando o vetor de estado não é disponível para medição, 
deve-se estudar o problema de rejeição de perturbações por realimentação de saída (DDPM, do inglês disturbance decoupling problem by measurement feedback). Este problema consiste em calcular um controlador por realimentação dinâmica da saída medida tal que a função de transferência das pertubações para a saída controlada seja igual a zero. O DDPM foi resolvido em (Schumacher, 1980; Willems e Commault, 1981).

Pouca atenção foi dada até o momento a um problema de formulação mais simples, o problema de rejeição de perturbações por realimentação estática de saída (DDPKM. Adota-se aqui o acrônimo adotado em (Chen, 1997) para Disturbance Decoupling Problem by Static Measurement Feedback). As condições de existência de soluções para o DDPKM foram inicialmente estabelecidas em termos da existência de um subespaço que goza de determinadas propriedades (Hamano e Furuta, 1975; Schumacher, 1980). Entretanto, é bastante difícil verificar a existência deste subespaço no caso geral.

Uma solução para o DDPKM para sistemas inversíveis à esquerda foi proposta recentemente em (Chen, 1997). O sistema é inicialmente representado em uma base de coordenadas especial na qual o DDPKM é solúvel se e somente se uma submatriz específica é nula.

Neste trabalho, os resultados de (Chen, 1997) relativos ao DDPKM são estendidos considerando-se um conjunto mais abrangente de sistemas. Para duas classes importantes de sistemas, estabelecem-se condições necessárias e suficientes para a existência de soluções. Diferentemente de (Chen, 1997), tais condições são expressas em termos geométricos, independentes da base de representação escolhida. Uma caracterização completa das soluções, bem como a parametrização de todas as matrizes de realimentação de saída correspondentes são também fornecidas.

\section{CONCEITOS E RESULTADOS PRELIMI- NARES}

Seja o sistema linear, de tempo contínuo, invariante no tempo, descrito por:

$$
\begin{gathered}
\dot{x}(t)=A x(t)+B u(t)+E q(t), \\
y(t)=C x(t), \\
z(t)=D x(t),
\end{gathered}
$$

em que $x \in \Re^{n}$ é o vetor de variáveis de estado, $u \in \Re^{m}$ é o vetor de entradas de controle, $q \in \Re^{d}$ é o vetor de entradas de perturbações, supostas não diretamente disponíveis para medição, $y \in \Re^{l}$ é o vetor de saídas medidas e $z \in \Re^{p}$ é o vetor de saídas a serem controladas. Supõe-se que as matrizes $B$ e $E$ possuem posto de coluna completo e que as matrizes $C$ e $D$ possuem posto de linha completo.
Os espaços imagem das matrizes $B$ e $E$ serão aqui denotados respectivamente por:

$$
\mathcal{B}=\operatorname{Im}(B), \quad \mathcal{E}=\operatorname{Im}(E),
$$

assim como os espaços nulos das matrizes $C$ e $D$ serão denotados respectivamente por:

$$
\mathcal{C}=\operatorname{ker}(C), \mathcal{K}=\operatorname{ker}(D)
$$

O objetivo do DDPKM é calcular, se possível, uma lei de controle por realimentação estática de saída, $u(t)=K y(t)$, tal que a matriz de transferência da perturbação $q$ para a saída controlada $z$ seja nula.

Dois conceitos são de fundamental importância no estudo deste problema, os de subespaços $(A, B)$-invariantes e $(C, A)$-invariantes (Wonham, 1985; Basile e Marro, 1992).

Definição 2.1 Considere-se o par $(A, B)$ relativo ao sistema (1). Um subespaço $\mathcal{V} \subset \Re^{n}$ é $(A, B)$-invariante se $A \mathcal{V} \subset$ $\mathcal{V}+\mathcal{B}$.

Pode-se mostrar que, equivalentemente, $\mathcal{V}$ é $(A, B)$ invariante se e somente se existe uma matriz $F \in \Re^{m \times n}$ tal que $(A+B F) \mathcal{V} \subset \mathcal{V}$.

Pode-se também mostrar a existência de um subespaço $(A, B)$-invariante de dimensão máxima entre todos aqueles contidos no subespaço $\mathcal{K}$ :

$$
\mathcal{V}^{*}=\begin{gathered}
\text { máximo subespaço }(A, B) \text {-invariante } \\
\text { contido em } \mathcal{K} .
\end{gathered}
$$

Definição 2.2 Considere-se o par $(C, A)$ relativo ao sistema (1), (2). Um subespaço $\mathcal{S} \subset \Re^{n}$ é $(C, A)$-invariante se $A(\mathcal{S} \cap \mathcal{C}) \subset \mathcal{S}$.

Equivalentemente, $\mathcal{S}$ é $(C, A)$-invariante se e somente se existe uma matriz $G \in \Re^{n \times l}$ tal que $(A+G C) \mathcal{S} \subset \mathcal{S}$.

O conjunto de todos os subespaços $(C, A)$-invariantes que contêm o subespaço $\mathcal{E}$ possui um elemento de dimensão mínima:

$$
\mathcal{S}_{*}=\begin{gathered}
\text { mínimo subespaço }(C, A) \text {-invariante } \\
\text { contendo } \mathcal{E} .
\end{gathered}
$$

As condições de existência de soluções para o DDPKM são dadas pelo seguinte teorema (Hamano e Furuta, 1975; Schumacher, 1980):

Teorema 2.1 O DDPKM é solúvel se e somente se existe um subespaço $\mathcal{V}$ que seja $(A, B)$ - e $(C, A)$-invariante e tal que $\mathcal{E} \subset \mathcal{V} \subset \mathcal{K}$. 
Equivalentemente, o DDPKM é solúvel se e somente se existe um subespaço $\mathcal{V}$ e uma matriz $K \in \Re^{m \times l}$ tais que $\mathcal{E} \subset \mathcal{V} \subset \mathcal{K}$ e $(A+B K C) \mathcal{V} \subset \mathcal{V}$

O teorema anterior estabelece condições necessárias e suficientes para a existência de realimentação estática de saída que resolve o DDPKM, em termos da existência de um subespaço que goza de determinadas propriedades. Porém, o mesmo não provê um método construtivo para verificar a existência deste subespaço. Trata-se de algo muito difícil no caso geral. De acordo com o que será mostrado na seqüência, se o sistema verifica determinadas condições, pode-se facilmente concluir sobre a existência de soluções para o DDPKM. Além disso, pode-se também facilmente obter a caracterização das soluções bem como a parametrização de todas as matrizes de realimentação de saída correspondentes.

\section{SOLUÇÃO PARA SISTEMAS SATISFA- ZENDO $\mathcal{B} \cap \mathcal{V}^{*} \subset \mathcal{S}_{*}$}

Definição 3.1 (Basile e Marro, 1982; Basile e Marro, 1992) Um subespaço $(A, B)$-invariante $\mathcal{V}$ contido em um subespaço $\mathcal{K}$ é dito auto-limitado ("self-bounded") em relação a $\mathcal{K}$ se $\mathcal{B} \cap \mathcal{V}^{*} \subset \mathcal{V}$.

Um subespaço $(A, B)$-invariante $\mathcal{V} \subset \mathcal{K}$ é auto-limitado em relação a $\mathcal{K}$ se para $x(0) \in \mathcal{V}$ o vetor de estado não pode deixar $\mathcal{V}$ através de trajetórias contidas em $\mathcal{K}$.

Uma caracterização detalhada de subespaços auto-limitados pode ser encontrada em (Basile e Marro, 1982; Basile e Marro, 1992). Entre as muitas propriedades de que gozam tais subespaços, pode-se destacar a seguinte (Basile e Marro, 1992):

Propriedade 3.1 O conjunto formado por todos os subespaços $(A, B)$-invariantes auto-limitados contidos em um subespaço $\mathcal{K}$ é fechado em relação à operação de interseção.

Considere-se agora a seguinte classe de subespaços:

$$
\mathcal{J}\left(A, B ; \mathcal{K}, \mathcal{S}_{*}\right) \triangleq\left\{\mathcal{V}: \mathcal{V} \in \mathcal{I}(A, B ; \mathcal{K}) \text { e } \mathcal{V} \supset \mathcal{S}_{*}\right\}
$$

em que $\mathcal{I}(A, B ; \mathcal{K})$ representa o conjunto de subespaços $(A, B)$-invariantes contidos em $\mathcal{K}$.

Note-se a partir do teorema 2.1 que uma condição necessária para existência de soluções ao DDPKM é que o conjunto $\mathcal{J}\left(A, B ; \mathcal{K}, \mathcal{S}_{*}\right)$ não seja vazio.

Supor-se-á na sequiência desta seção que o sistema em estudo satisfaz a condição:

$$
\mathcal{B} \cap \mathcal{V}^{*} \subset \mathcal{S}_{*}
$$

Uma categoria importante de sistemas que verificam esta condição é aquela dos sistemas cuja matriz de transferência do trio $(A, B, D)$, é inversível à esquerda. Esta propriedade implica a possibilidade de se reconstruir a entrada $u(t)$ através da saída $z(t)$.

Sistemas inversíveis à esquerda são caracterizados geometricamente pela condição (Wonham, 1985; Basile e Marro, 1992): $\mathcal{B} \cap \mathcal{V}^{*}=0$. Em (Chen, 1997), o DDPKM é estudado unicamente para este tipo de sistema.

Deve-se ressaltar que, sendo os sistemas aqui considerados estritamente próprios, a inversa da matriz de transferência pode possuir termos impróprios. Este caso pode indistintamente ser tradado através da abordagem aqui proposta, visto que esta inversa não é de nenhum modo utilizada.

Lema 3.1 Se $\mathcal{B} \cap \mathcal{V}^{*} \subset \mathcal{S}_{*}$, então $\mathcal{J}\left(A, B ; \mathcal{K}, \mathcal{S}_{*}\right)$ possui um elemento de dimensão mínima:

$$
\mathcal{V}_{*}=\min \mathcal{V} \in \mathcal{J}\left(A, B ; \mathcal{K}, \mathcal{S}_{*}\right) \text {. }
$$

Demonstração: Basta mostrar que $\mathcal{J}\left(A, B ; \mathcal{K}, \mathcal{S}_{*}\right)$ é fechado em relação à operação de interseção (Wonham, 1985; Basile e Marro, 1992). Para $\mathcal{V} \in \mathcal{J}\left(A, B ; \mathcal{K}, \mathcal{S}_{*}\right), \mathcal{B} \cap$ $\mathcal{V}^{*} \subset \mathcal{S}_{*} \subset \mathcal{V}, \operatorname{logo} \mathcal{V}$ é um subespaço $(A, B)$-invariante auto-limitado e, a partir da propriedade 3.1, o conjunto $\mathcal{J}\left(A, B ; \mathcal{K}, \mathcal{S}_{*}\right)$ é fechado em relação à operação de interseção.

O subespaço $\mathcal{V}_{*}$ pode ser caracterizado da seguinte forma:

Teorema 3.1 (Basile e Marro, 1992) Seja $\mathcal{U}_{*}$ o mínimo subespaço $(D, A)$-invariante que contém $\mathcal{B}$. Então $\mathcal{V}_{*}=\mathcal{V}^{*} \cap$ $\mathcal{U}_{*}$.

Considere-se agora o seguinte conjunto de matrizes de realimentação de estado:

$$
\mathbf{F}(\mathcal{V}) \triangleq\left\{F \in \Re^{m \times n}:(A+B F) \mathcal{V} \subset \mathcal{V}\right\} .
$$

Lema 3.2

$$
\mathbf{F}\left(\mathcal{V}_{*}\right) \supset \mathbf{F}(\mathcal{V}) \forall \mathcal{V} \in \mathcal{J}\left(A, B ; \mathcal{K}, \mathcal{S}_{*}\right)
$$

Demonstração: Basta mostrar que qualquer matriz $F \in$ $\mathbf{F}(\mathcal{V})$, com $\mathcal{V} \in \mathcal{J}\left(A, B ; \mathcal{K}, \mathcal{S}_{*}\right)$ pertence também a $\mathbf{F}\left(\mathcal{V}_{*}\right)$. Por definição, $\mathcal{V}_{*}$ é tal que:

$$
A \mathcal{V}_{*} \subset \mathcal{V}_{*}+\mathcal{B}, \mathcal{B} \cap \mathcal{V}^{*} \subset \mathcal{V}_{*}, \mathcal{V}_{*} \subset \mathcal{V}
$$

Qualquer $F \in \mathbf{F}(\mathcal{V})$ é tal que:

$$
(A+B F) \mathcal{V} \subset \mathcal{V} .
$$


Assim,

$$
(A+B F) \mathcal{V}_{*} \subset(A+B F) \mathcal{V} \subset \mathcal{V}
$$

Além disso, como $\mathcal{V}_{*} e ́(A, B)$-invariante e $B F \mathcal{V}_{*} \subset \mathcal{B}$,

$$
(A+B F) \mathcal{V}_{*} \subset A \mathcal{V}_{*}+\mathcal{B} \subset \mathcal{V}_{*}+\mathcal{B}
$$

A partir das relações (4), (5) acima, conclui-se que:

$$
\begin{aligned}
(A+B F) \mathcal{V}_{*} & \subset \mathcal{V} \cap\left(\mathcal{V}_{*}+\mathcal{B}\right) \\
& \subset \mathcal{V}_{*}+\mathcal{B} \cap \mathcal{V} \\
& \subset \mathcal{V}_{*}+\mathcal{B} \cap \mathcal{V}^{*} \\
& \subset \mathcal{V}_{*} .
\end{aligned}
$$

Os resultados anteriores permitem estabelecer condições de existência de soluções ao DDPKM para a família de sistemas em questão.

Teorema 3.2 Para sistemas em que $\mathcal{B} \cap \mathcal{V}^{*} \subset \mathcal{S}_{*}$, o DDPKM é solúvel se e somente se $\mathcal{V}_{*}$ é um subespaço $(C, A)$ invariante.

\section{Demonstração:}

Necessidade: Se o DDPKM é solúvel, então existe uma matriz $K \in \Re^{m \times l}$ e um subespaço $\mathcal{V}$ tais que $\mathcal{S}_{*} \subset \mathcal{V} \subset$ $\mathcal{V}^{*} e(A+B K C) \mathcal{V} \subset \mathcal{V}$. Isto garante que o conjunto $\mathcal{J}\left(A, B ; \mathcal{K}, \mathcal{S}_{*}\right)$ não é vazio e, conseqüentemente, a existência de $\mathcal{V}_{*}$. Além disso, em virtude do lema 3.2, tem-se que $(A+B K C) \mathcal{V}_{*} \subset \mathcal{V}_{*}$, portanto $\mathcal{V}_{*} e ́(C, A)$-invariante.

Suficiência: $S e \mathcal{V}_{*} e ́(C, A)$-invariante, já que $\mathcal{V}_{*}$ é também $(A, B)$-invariante e por definição $\mathcal{E} \subset \mathcal{V}_{*} \subset \mathcal{K}$, de acordo com o teorema 2.1 o DDPKM deve ser solúvel.

Ressalte-se que as condições geométricas estabelecidas por este teorema independem da base escolhida para representar os espaços de estado, entrada e saída do sistema. Além disso, diferentemente das condições propostas em (Chen, 1997), aplicam-se não apenas a sistemas inversíveis à esquerda, mas a uma família bem mais ampla.

Na próxima seção, condições semelhantes são obtidas para outra família importante de sistemas.

\section{SOLUÇÃO PARA SISTEMAS SATISFA- ZENDO $\mathcal{V}^{*} \subset \mathcal{C}+\mathcal{S}_{*}$}

A condição geométrica que define a classe de sistemas objeto desta seção é dual àquela da seção anterior. Os resultados apresentados a seguir podem também ser obtidos facilmente por dualidade. Assim, visando a evitar repetições desnecessárias, suas demonstrações serão omitidas.

Definição 4.1 (Basile e Marro, 1992) Um subespaço $(C, A)$-invariante $\mathcal{S}$ que contém um subespaço $\mathcal{E}$ é dito autooculto ("self-hidden") em relação a $\mathcal{E}$ se $\mathcal{S} \subset \mathcal{S}_{*}+\mathcal{C}$.
Um subespaço $(C, A)$-invariante $\mathcal{S} \supset \mathcal{E}$ é auto-oculto em relação a $\mathcal{E}$ se o mesmo pode tornar-se não observável com um observador dinâmico na presença de perturbações pertencentes a $\mathcal{E}$.

Uma propriedade importante de subespaços auto-ocultos é destacada a seguir (Basile e Marro, 1992):

Propriedade 4.1 O conjunto formado por todos os subespaços $(C, A)$-invariantes auto-ocultos que contêm um subespaço $\mathcal{E}$ é fechado em relação à operação de soma de subespaços.

Considere-se agora a seguinte classe de subespaços:

$$
\mathcal{J}\left(C, A ; \mathcal{E}, \mathcal{V}^{*}\right) \triangleq\left\{\mathcal{S}: \mathcal{S} \in \mathcal{I}(C, A ; \mathcal{E}) \text { e } \mathcal{S} \subset \mathcal{V}^{*}\right\}
$$

em que $\mathcal{I}(C, A ; \mathcal{E})$ representa o conjunto de subespaços $(C, A)$-invariantes que contêm $\mathcal{E}$.

Note-se a partir do teorema 2.1 que uma condição necessária para existência de soluções ao DDPKM é que o conjunto $\mathcal{J}\left(C, A ; \mathcal{E}, \mathcal{V}^{*}\right)$ não seja vazio.

Supor-se-á na seqüência desta seção que o sistema em estudo satisfaz a condição:

$$
\mathcal{V}^{*} \subset \mathcal{C}+\mathcal{S}_{*}
$$

Uma categoria importante de sistemas que verificam esta condição é aquela dos sistemas cuja matriz de transferência do trio $(A, E, C)$, é inversível à direita. Isto implica a possibilidade de se impor qualquer saída $y(t)$, bem comportada, através de uma entrada $q(t)$ conveniente (controlabilidade funcional). Também neste caso, a possibilidade da inversa da matriz de transferência conter elementos impróprios não tem nenhuma implicação sobre os resultados aqui relatados.

Trios $(A, E, C)$ inversíveis à direita são caracterizados geometricamente pela condição (Basile e Marro, 1992): $\mathcal{C}+$ $\mathcal{S}_{*}=\Re^{n}$.

Lema 4.1 $\operatorname{Se} \mathcal{V}^{*} \subset \mathcal{C}+\mathcal{S}_{*}$, então $\mathcal{J}\left(C, A ; \mathcal{E}, \mathcal{V}^{*}\right)$ possui um elemento de dimensão máxima:

$$
\mathcal{S}^{*}=\max \mathcal{S} \in \mathcal{J}\left(C, A ; \mathcal{E}, \mathcal{V}^{*}\right) \text {. }
$$

Demonstração: Basta mostrar que $\mathcal{J}\left(C, A ; \mathcal{E}, \mathcal{V}^{*}\right)$ é fechado em relação à operação de adição de subespaços (Wonham, 1985; Basile e Marro, 1992). Para $\mathcal{V} \in$ $\mathcal{J}\left(C, A ; \mathcal{E}, \mathcal{V}^{*}\right), \mathcal{C}+\mathcal{S}_{*} \supset \mathcal{V}^{*} \supset \mathcal{V} \supset \mathcal{E}, \operatorname{logo} \mathcal{V}$ é um subespaço $(A, B)$-invariante auto-oculto $e$, a partir da propriedade 4.1 , o conjunto $\mathcal{J}\left(C, A ; \mathcal{E}, \mathcal{V}^{*}\right)$ é fechado em relação à operação de adição de subespaços. 
O subespaço $\mathcal{S}^{*}$ pode ser caracterizado da seguinte forma:

Teorema 4.1 (Basile e Marro, 1992) Seja $\mathcal{W}^{*}$ o máximo subespaço $(A, E)$-invariante contido em $\mathcal{C}$. Então $\mathcal{S}^{*}=$ $\mathcal{S}_{*}+\mathcal{W}^{*}$.

Condições necessárias e suficentes para exis-tência de soluções ao DDPKM para a família de sistemas em estudo são dadas pelo seguinte teorema:

Teorema 4.2 Para sistemas para os quais $\mathcal{V}^{*} \subset \mathcal{C}+\mathcal{S}_{*}$, o DDPKM é solúvel se e somente se $\mathcal{S}^{*}$ é um subespaço $(A, B)$-invariante.

\section{PARAMETRIZAÇÃO DAS SOLUÇÕES}

A partir dos teoremas 3.1 e 4.1 , nota-se que $\mathcal{V}_{*}$ e $\mathcal{S}^{*}$ podem ser obtidos a partir dos cálculos de $\mathcal{V}^{*}, \mathcal{U}_{*}, \mathcal{S}_{*}$ e $\mathcal{W}^{*}$, para os quais algoritmos eficientes e numericamente estáveis são disponíveis (Van Dooren, 1981; Dórea e Milani, 1995). Em particular, se uma das condições $B \cap \mathcal{V}^{*} \subset \mathcal{S}_{*}$ ou $\mathcal{V}^{*} \subset \mathcal{C}+\mathcal{S}_{*}$ é verificada, então é sempre possível determinar mudanças de base ortogonais tais que o sistema possa ser representado pelas seguintes matrizes:

$$
\begin{gathered}
A=\left[\begin{array}{ll}
A_{11} & A_{12} \\
A_{21} & A_{22}
\end{array}\right], B=\left[\begin{array}{cc}
B_{11} & B_{12} \\
B_{21} & 0
\end{array}\right], E=\left[\begin{array}{c}
E_{1} \\
0
\end{array}\right], \\
C=\left[\begin{array}{cc}
C_{11} & C_{12} \\
0 & C_{22}
\end{array}\right], D=\left[\begin{array}{ll}
0 & D_{2}
\end{array}\right],
\end{gathered}
$$

em que $B_{21}$ possui posto de coluna completo, $C_{11}$ possui posto de linha completo e

$$
\mathcal{V}_{*}=\operatorname{Im}\left[\begin{array}{l}
I \\
0
\end{array}\right] \quad\left(\operatorname{se} \mathcal{B} \cap \mathcal{V}^{*} \subset \mathcal{S}_{*}\right)
$$

ou

$$
\mathcal{S}^{*}=\operatorname{Im}\left[\begin{array}{l}
I \\
0
\end{array}\right] \quad\left(\operatorname{se} \mathcal{V}^{*} \subset \mathcal{C}+\mathcal{S}_{*}\right),
$$

em que 0 e $I$ representam respectivamente a matriz nula e a matriz identidade de dimensões apropriadas.

Note-se que as estruturas das matrizes $D$ e $E$ acima são obtidas supondo-se que a condição necessária para a rejeição de perturbações, $D E=0$ (Wonham, 1985), seja satisfeita.

A partir de agora, será considerado o primeiro caso $\left(\mathcal{B} \cap \mathcal{V}^{*} \subset\right.$ $\mathcal{S}_{*}$ ). Para o segundo caso $\left(\mathcal{V}^{*} \subset \mathcal{C}+\mathcal{S}_{*}\right)$, resultados semelhantes aos apresentados na seqüência podem ser obtidos por dualidade.

Isto posto, a representação acima reflete as seguintes decomposições dos espaços de estado, entrada e saída medida:

$$
\mathcal{X}=\mathcal{X}_{1} \oplus \mathcal{X}_{2}
$$

$$
\begin{aligned}
& \mathcal{U}=\mathcal{U}_{1} \oplus \mathcal{U}_{2}, \\
& \mathcal{Y}=\mathcal{Y}_{1} \oplus \mathcal{Y}_{2},
\end{aligned}
$$

em que $\mathcal{X}_{1}=\mathcal{V}_{*} ; B \mathcal{U}_{2}=\mathcal{B} \cap \mathcal{V}^{*}=\mathcal{B} \cap \mathcal{V}_{*}, B \mathcal{U}=\mathcal{B}$; $C \mathcal{X}=\mathcal{Y}, C_{2} \mathcal{X}=\mathcal{Y}_{2}, \operatorname{ker}\left(C_{2}\right)=\mathcal{C}+\mathcal{V}_{*}$.

Proposição 5.1 Para sistemas em que $\mathcal{B} \cap \mathcal{V}^{*} \subset \mathcal{S}_{*}$, o DDPKM é solúvel se e somente se existe uma matriz $K_{11}$ tal que:

$$
A_{21}+B_{21} K_{11} C_{11}=0 .
$$

Neste caso, o conjunto de todas as matrizes de realimentação estática de saída que resolvem o DDPKM é dado por:

$$
K=\left[\begin{array}{ll}
K_{11} & K_{12} \\
K_{21} & K_{22}
\end{array}\right]
$$

em que $K_{11}$ é dado pela solução única dos sistemas de equações lineares (8) e $K_{12}, K_{21}$ e $K_{22}$ são livres para assumir qualquer valor.

Demonstração: De acordo com o teorema 3.2, basta verificar se o subespaço $(A, B)$-invariante $\mathcal{V}_{*}=\operatorname{Im}\left[\begin{array}{l}I \\ 0\end{array}\right]$ é também $(C, A)$-invariante, ou seja, se existe uma matriz $K$ (9) tal que $(A+B K C) \mathcal{V}_{*} \subset \mathcal{V}_{*}$. Constata-se por inspeção que isto é possível se e somente se (8) é satisfeita.

Além disso, a partir do lema 3.2, conclui-se que o conjunto de todos os produtos de matrizes $K C$ que resolvem o DDPKM é dado por $K C \in \mathbf{F}\left(\mathcal{V}_{*}\right)$. Considerando-se

$$
F=\left[\begin{array}{ll}
F_{11} & F_{12} \\
F_{21} & F_{22}
\end{array}\right],
$$

tem-se que (Dórea e Milani, 1997):

$$
\mathbf{F}\left(\mathcal{V}_{*}\right)=\left\{F: A_{21}+B_{21} F_{11}=0\right\} .
$$

Assim, com $K$ dado por (9) e em virtude de $C_{11}$ ter posto de linha completo, pode-se concluir que a submatriz $K_{11}$ deve ser a solução única de $K_{11} C_{11}=F_{11}$ e portanto também da equação (8). Os valores das outras submatrizes compondo $K$ são claramente irrelevantes.

A parametrização acima explicita os graus de liberdade da matriz de realimentação de saída $K$, possibilitando assim a utilização destes na satisfação de outros objetivos de controle além da rejeição de perturbações.

Um desses objetivos é o de estabilidade do sistema em malha fechada. É fácil verificar que com a parametrização acima, este problema reduz-se ao problema de estabilização por realimentação estática de saída dos trios $\left(A_{11}+\right.$ $\left.B_{11} K_{11} C_{11}, B_{12}, C_{11}\right)$ e $\left(A_{22}+B_{21} K_{11} C_{12}, B_{21}, C_{22}\right)$. Trata-se ainda de um problema em aberto, para cuja solução entretanto diversas técnicas numéricas baseadas em 
condições suficientes são disponíveis (Syrmos et al., 1997). Conseguindo-se estabilizar o sistema, é ainda possível utilizar os graus de liberdade da solução do DDPKM para otimizar um índice de desempenho quadrático, como em (Dórea e Milani, 1995).

\section{EXEMPLOS NUMÉRICOS}

Exemplo 1 Seja o sistema (1), (3) para o qual:

$$
\begin{gathered}
A=\left[\begin{array}{ccccc}
-2 & 1 & -1 & 0 & 0 \\
-2 & -1 & -1 & -1 & -1 \\
6 & -1 & 5 & -1 & 0 \\
6 & -2 & 5 & -1 & 1 \\
2 & 4 & 1 & 2 & 0
\end{array}\right], \quad B=\left[\begin{array}{cc}
-2 & -1 \\
-1 & 0 \\
1 & 0 \\
3 & 1 \\
1 & 0
\end{array}\right], \\
E=\left[\begin{array}{cc}
-1 & 0 \\
-3 & 2 \\
4 & -2 \\
5 & -2 \\
2 & -2
\end{array}\right], \quad D=\left[\begin{array}{llll}
0 & 1 & -1 & 1
\end{array}\right]
\end{gathered}
$$

A solução do problema de rejeição de perturbações por realimentação de estado para este sistema foi estudada em (Dórea e Milani, 1995; Dórea e Milani, 1997). Pode-se verificar que o sistema representado por estas matrizes satisfazem a condição $\mathcal{B} \cap \mathcal{V}^{*} \subset \mathcal{S}_{*}$.

Suponhamos agora que não se disponha do acesso completo a suas variáveis de estado, mas apenas a um conjunto de saídas medidas representado pela equação (2), em que:

$$
C=\left[\begin{array}{ccccc}
0 & 0 & 1 & 0 & 0 \\
0 & 1 & 0 & -1 & 0 \\
1 & 2 & 1 & 0 & 1
\end{array}\right] \text {. }
$$

Aplicando-se ao sistema as seguintes mudanças de variáveis:

$$
\begin{aligned}
& \bar{x}=Q^{T} x, \\
& \bar{u}=Z_{B}^{T} u, \\
& \bar{y}=Z_{C}^{T} y,
\end{aligned}
$$

com as matrizes ortogonais $Q, Z_{B}$ e $Z_{C}$ dadas por:

$$
\begin{gathered}
Q=\left[\begin{array}{ccccc}
0.2700 & -0.3062 & -0.9065 & 0.1080 & 0 \\
0.2400 & 0.4838 & -0.1700 & -0.6553 & 0.5 \\
-0.5100 & -0.1776 & -0.1700 & -0.6553 & -0.5 \\
-0.7800 & 0.1286 & -0.2455 & 0.2544 & 0.5 \\
0.0300 & -0.7900 & 0.2455 & -0.2544 & 0.5
\end{array}\right], \\
Z_{B}=\left[\begin{array}{ccc}
-0.8944 & -0.4472 \\
-0.4472 & 0.8944
\end{array}\right],
\end{gathered}
$$

$$
Z_{C}=\left[\begin{array}{ccc}
-0.4352 & 0.1030 & 0.8944 \\
0.8704 & -0.2061 & 0.4472 \\
0.2304 & 0.9731 & 0
\end{array}\right],
$$

as matrizes que o representam tomam a forma das equações (6), (7):

$$
\begin{gathered}
\bar{A}=\left[\begin{array}{cc|ccc}
0.8214 & 5.9177 & 8.1642 & 2.6890 & 4.6204 \\
0.6105 & -1.0297 & 3.3197 & 2.7724 & -2.5933 \\
\hline-0.1983 & 0.7245 & -0.4453 & 0.2105 & 1.3315 \\
-0.0439 & 0.1605 & 2.0476 & 0.9037 & 1.5303 \\
-0.1500 & 0.5481 & -0.2927 & -0.5281 & 0.7500
\end{array}\right], \\
\bar{B}=\left[\begin{array}{cc|cc}
3.6897 & 0.6708 \\
0.2111 & 0.5916 \\
\hline-1.4780 & 0 \\
-0.3275 & 0 \\
-1.1180 & 0
\end{array}\right], \quad \bar{E}=\left[\begin{array}{ccc}
-6.8702 & 3 \\
-2.7929 & 2.6458 \\
0 & 0 \\
0 & 0 \\
0 & 0
\end{array}\right], \\
\bar{C}=\left[\begin{array}{cc|cc}
1.1720 & 0.3159 & -0.1301-0.9933 & 0.4480 \\
0 & -0.3895 & -1.1725-1.9356 & 0.9216 \\
\hline 0.0000 & 0.0000 & -0.1183-0.9930 & -0.4472
\end{array}\right],
\end{gathered}
$$

Nesta representação,

$$
\overline{\mathcal{V}}^{*}=\operatorname{ker}(\bar{D})=\operatorname{Im}\left[\begin{array}{c}
I_{4} \\
0
\end{array}\right],
$$

$$
\begin{gathered}
\overline{\mathcal{B}} \cap \overline{\mathcal{V}}^{*}=\operatorname{Im}\left[\begin{array}{c}
0.6708 \\
0.5916 \\
0 \\
0 \\
0
\end{array}\right] \subset \operatorname{Im}(\bar{E}) \subset \overline{\mathcal{V}}^{*}, \\
\overline{\mathcal{V}}_{*}=\operatorname{Im}\left[\begin{array}{c}
I_{2} \\
0
\end{array}\right],
\end{gathered}
$$

em que $I_{q}$ representa a matriz identidade de ordem $q$.

Pode-se verificar por inspeção que $\overline{\mathcal{V}}_{*}$ é $(C, A)$-invariante. Portanto, de acordo com o teorema 3.2, o DDPKM é solúvel. A partir da proposição 5.1, o conjunto de todas as matrizes de realimentação de saída que resolvem o DDPKM é parametrizado por:

$$
\bar{K}=\left[\begin{array}{cc|c}
-0.1145 & -1.3516 & X \\
\hline X & X & X
\end{array}\right]
$$

em que os elementos " $X$ " são livres para assumir qualquer valor.

Exemplo 2 Seja o sistema massa-mola-amortecedor na Figura 1 , em que $x_{1}, x_{2}$ são deslocamentos relativos ao ponto de equilíbrio e $q(t), u(t)$ são forças. 
Tomando-se como vetor de estado

$$
x(t)=\left[\begin{array}{c}
x_{1} \\
\dot{x}_{1} \\
x_{2} \\
\dot{x}_{2}
\end{array}\right]
$$

e assumindo $u(t), q(t)$ e $z(t)=x_{2}$ como vetores de variáveis de controle, perturbação e saída a ser controlada, respectivamente, obtém-se a seguinte representação para o sistema:

$$
\begin{gathered}
\dot{x}(t)=A x(t)+B u(t)+E q(t), \\
z(t)=D x(t),
\end{gathered}
$$

em que:

$$
\begin{aligned}
& A=\left[\begin{array}{cccc}
0 & 1 & 0 & 0 \\
-\left(k_{1}+k_{2}\right) / m_{1} & -c_{1} / m_{1} & k_{2} / m_{1} & 0 \\
0 & 0 & 0 & 1 \\
k_{2} / m_{2} & 0 & -k_{2} / m_{2} & 0
\end{array}\right] \text {, } \\
& B=\left[\begin{array}{c}
0 \\
0 \\
0 \\
1 / m_{2}
\end{array}\right], \quad E=\left[\begin{array}{c}
0 \\
1 / m_{1} \\
0 \\
0
\end{array}\right] \\
& D=\left[\begin{array}{llll}
0 & 0 & 1 & 0
\end{array}\right] \text {. }
\end{aligned}
$$

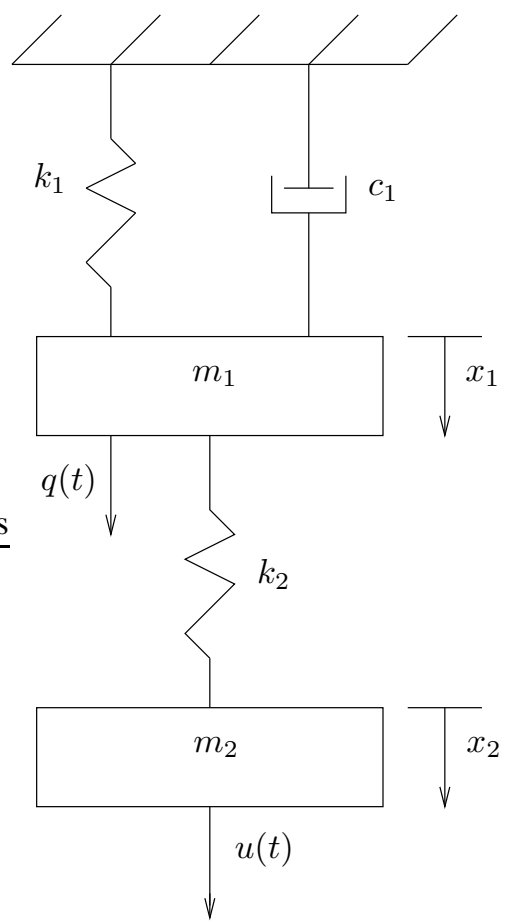

Figura 1: Sistema massa-mola-amortecedor
Adotando como variável de saída medida

$$
y(t)=C x(t), \quad C=\left[\begin{array}{llll}
1 & 0 & 0 & 1
\end{array}\right],
$$

deseja-se calcular um controlador por realimentação de saída, $u(t)=K y(t)$ de modo que o efeito de $q(t)$ não seja sentido na saída controlada $z(t)$.

Pode ser verificado que $\mathcal{V}^{*}=\mathcal{S}_{*}=\operatorname{Im}\left[\begin{array}{c}I_{2} \\ 0\end{array}\right]$. Deste modo, facilmente constata-se que $\mathcal{B} \cap \mathcal{V}^{*}=0$ e $\mathcal{S}_{*}+\mathcal{C}=\Re^{4}$, ou seja, o trio $(A, B, D)$ é inversível à esquerda e o trio $(A, E, C)$ é inversível à direita. Além disso, $\mathcal{V}_{*}=\mathcal{V}^{*}$, que, por sua vez é $(C, A)$-invariante. De fato, estes subespaços podem ser feitos $(A+B K C)$-invariantes através da lei de controle $u(t)=-k_{2} y(t)$.

Em malha fechada:

$\dot{x}(t)=\left[\begin{array}{cccc}0 & 1 & 0 & 0 \\ -\left(k_{1}+k_{2}\right) / m_{1} & -c_{1} / m_{1} & k_{2} / m_{1} & 0 \\ 0 & 0 & 0 & 1 \\ 0 & 0 & -k_{2} / m_{2}-k_{2} / m_{2}\end{array}\right] x(t)$

Portanto, o controlador rejeita o efeito da perturbação na saída e torna o sistema em malha fechada assintoticamente estável.

\section{CONCLUSÃO}

Pouca atenção tinha sido dada até há bem pouco tempo ao estudo do problema de rejeição de perturbações por realimentação estática de saída. Isto se deve certamente à dificuldade em se estabelecerem condições de existência de soluções. Mostrou-se aqui que se o sistema verifica certas propriedades, então é possível não somente concluir sobre a existência de soluções como também parametrizar o conjunto de matrizes associadas. Condições geométricas necessárias e suficientes foram então estabelecidas. Categorias importantes de sistemas verificam as propriedades anteriormente citadas, entre os quais os sistemas inversíveis à esquerda e à direita. Este estudo estende assim resultados recentemente publicados na literatura, restritos a sistemas inversíveis à esquerda, com condições de existência de soluções estabelecidas em relação a uma base particular de representação do sistema.

\section{REFERÊNCIAS}

Basile, G. e Marro, G. (1982). Self-bounded controlled invariant subspaces: a straightforward approach to constrained controllability, J. Optimiz. Theory Appl. 38: 71-81.

Basile, G. e Marro, G. (1992). Controlled and Conditioned Invariants in Linear System Theory, Prentice-Hall. 
Chen, B. M. (1997). Solvability conditions for disturbance decoupling problems with static measurement feedback, Int. J. Contr. 68: 51-60.

Dórea, C. E. T. e Milani, B. E. A. (1995). A computational method for optimal L-Q regulation with simultaneous disturbance decoupling, Automatica 31(1): 155-160.

Dórea, C. E. T. e Milani, B. E. A. (1997). Disturbance decoupling in a class of linear systems, IEEE Trans. Automat. Contr. 42(10): 1427-1431.

Hamano, F. e Furuta, K. (1975). Localization of disturbances and output decomposition in decentralized linear multivariable systems, Int. J. Contr. 22: 551-562.

Schumacher, J. M. (1980). Compensator synthesis using $(C, A, B)$-pairs, IEEE Trans. Automat. Contr. 25(6): 1133-1138.

Syrmos, V. L., Abdallah, C. T., Dorato, P. e Grigoriadis, K. (1997). Static output feedback - a survey, Automatica 33(2): 125-137.

Van Dooren, P. (1981). The generalized eigenstructure problem in linear system theory, IEEE Trans. Automat. Contr. 26: 111-129.

Willems, J. C. e Commault, C. (1981). Disturbance decoupling by measurement feedback with stability or pole placement, SIAM J. Contr. Optimiz. 19(4): 491-504.

Wonham, W. M. (1985). Linear Multivariable Control - A Geometric Approach, Springer-Verlag, New York. 